\title{
OPTICAL ERRORS IN A LIQUID CHROMATOGRAPHY ABSORBANCE CELL
}

\author{
KONAN PECK ${ }^{\star}$ and MICHAEL D. MORRIS*
}

Department of Chemistry, University of Michigan, Ann Arbor, MI 48109 (U.S.A.)

(Received April 19th, 1988)

\section{SUMMARY}

The shadowgraph method is used to visualize mobile phase flow through a liquid chromatography absorbance cell with " $Z$ " flow geometry. It is shown that mixing is incomplete in the cell. Composition gradients persist throughout the elution of any sample. The gradients systematically change as the sample flows through the cell. Additionally, window wedge angles are measured in several cells. The implications for low noise absorbance detectors are discussed.

\section{INTRODUCTION}

The detection method most widely used in liquid chromatography (LC) ${ }^{1,2}$ is ultraviolet-visible (UV-VIS) absorption. The sensitivity of most absorption spectroscopic measurements is limited by intensity fluctuations caused by various experimental problems, and is usually well above the electronic shot noise limit. Modern mercury, zinc, or cadmium lamps are sufficiently intense to yield shot noise limits equivalent to $10^{-7}$ absorbance unit (a.u.). With modern deuterium lamps, the shot noise limit is about $10^{-6}$ a.u. But the current detection limits for absorbance measurements are in the order of $10^{-5}$ a.u. Therefore, it is important to identify and eliminate or compensate for excess noise sources.

One common noise source is mobile phase impurities which absorb at detection wavelengths. These impurities are present at relatively high levels in commercial solvents and in other chemicals used as components of the mobile phase. Therefore, careful purification of the solvents or the use of chromatographic-grade solvents are necessary to achieve good sensitivity.

Another noise source is fluctuation of mobile phase refractive index during measurement. Commonly, this arises from temperature and composition gradients in the mobile phase. The temperature gradient causes a refractive index variation, which in turn appears as noise in the absorption measurement. To achieve shot noise detection limit on the order of $10^{-7}$ a.u. requires controlling the temperature of the mobile phase to $\pm 0.004^{\circ} \mathrm{C}$ at $195 \mathrm{~nm}$ or $\pm 0.4^{\circ} \mathrm{C}$ at $254 \mathrm{~nm}$ (ref. 2). Such precise control would require much more sophisticated flow cell designs and thermostatting than are

\footnotetext{
^ Present address: Department of Chemistry, University of California, Berkeley, CA 94720, U.S.A.
} 
currently employed. In addition, a refractive index change can also be caused by variations in mobile phase flow-rate or pressure, and solvent gradients due to incompletely mixed solvents. The solvent gradient profile itself in gradient elution LC is another noise source ${ }^{3}$.

The design of the flow cell and the properties of a photodiode contribute to excess noise. Any change in refractive index at the window of the flow cell or within the cell itself causes light rays in the cell to be refracted. Some of the light which would normally pass directly through the cell strikes the cell wall instead and is lost. The detector cannot distinguish light lost by sample absorption from light lost to cell walls.

To prevent the light from hitting the cell wall, Little and Fallick ${ }^{4}$ designed a cell with diverging walls to accommodate much of the refraction. In principle, with this configuration all of the light which enters the cell should leave the cell, unless there is true absorption. More recent studies of chromatographic flow cells have been aimed at maximizing the light throughput in a small working volume ${ }^{5,6}$.

But even the light rays that do not hit the cell wall are deflected from one point on the photodiode to another by refractive index gradients. Most photodiodes have inhomogeneity in spatial response inversely proportional to the illuminated area. For a $0.13-\mathrm{mm}$ spot, the response inhomogeneity may be as much as $1 \%$ (ref. 7 ). To the extent that the mobile phase is uniform and at constant temperature and pressure, there are no refractive index gradients when pure mobile phase is flowing. But, in gradient elution measurements or in high-sensitivity isocratic measurements, deflection itself becomes another noise sources which may increase detection limits. While sample is flowing through the cell, deflection can generate small systematic errors in measured absorbance.

A typical chromatographic flow cell has a tapered design and a Z-shaped flow path. The Z-path is used to minimize stagnant regions in the cell and to reduce peak tailing ${ }^{4}$. Variants on this design have largely replaced straight-through capillaries or $\mathrm{U}$ shaped flow paths in commercial LC instruments. Typically, the flow is confined by two quartz windows held in place by caps screwed though or into the cell body. Mobile phase flows in from the bottom of the cell, hits the cell window and is directed through the body of the cell, which is aligned along the light path. The flow hits the window on the other side of the cell and then is forced out of the top of the cell.

The design minimizes stagnant regions, but introduces new problems. First, it is easy to assemble the cell so that the windows are wedged, deflecting the entire light beam. However, unless the screws used to hold the cell windows in place are carefully adjusted, the cell windows will usually be wedged with respect to the cell body. More importantly, the $\mathrm{Z}$ path itself leads to complicated time-dependent mixing patterns in the cell.

A wedged cell behaves like a prism ${ }^{8}$, its influence can be seen from eqn. 1 and Fig. 1.

$$
\delta=\theta_{\mathrm{i}}+\sin ^{-1}\left[(\sin \alpha)\left(n^{2}-\sin ^{2} \theta_{\mathrm{i}}\right)^{1 / 2}-\sin \theta_{\mathrm{i}} \cos \alpha\right]-\alpha
$$

where $n$ is the refractive index. Fig. 1 shows the deviation angle, $\delta$, versus incident angle, $\theta_{\mathrm{i}}$, for sample cell wedged at two different angles, $\alpha$. It is clear from the graphs that at different incident angles the curves have different slopes. The incident angle can vary by improper assembly or mounting of the cell or even by prolonged operation. 

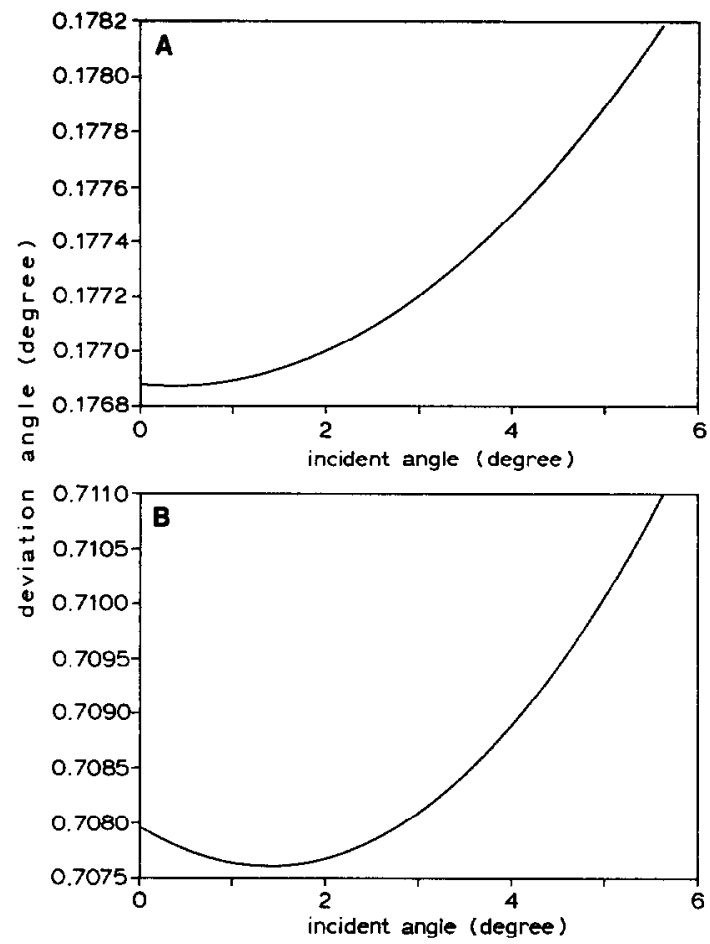

Fig. 1. Effect of cell angle on beam deviation in a wedged cell. (A) 9 mrad wedge angle; (B) lower graph 36 mrad wedge angle.

The temperature of the assembly may vary during operation because of heat dissipated from the electronic circuits or by the illumination with the light source. The temperature change can cause the beam to wander.

It is useful to observe these effects in a chromatographic flow cell in order to estimate their importance and to guide further designs. Flow visualization is an important experimental tool of fluid mechanics ${ }^{9}$. The range of flow visualization techniques is broad, but these techniques have been developed for observation of flow in or around large structures such as chemical reactor pipes or automobiles. At least three flow visualization techniques can be adapted to the scale of a LC flow cell. While the general optical principles remain unchanged, special techniques are required to observe refractive index gradients in a cell whose diameter is about $1 \mathrm{~mm}$.

Mach-Zehnder interferometry is based upon the interference of object light passing through the flow cell and reference light in a stationary medium to create a visible intensity distribution. The Mach-Zchnder interferometer measures the change of refractive index $\partial n$ or the distribution of refractive index directly. In principle, it produces a more direct measurement than the more common schlieren or shadow methods which measure $\partial n / \partial x$ and $\partial^{2} n / \partial x^{2}$ respectively.

The schlieren method measures directly refractive index gradients, using a knife edge as a spatial filter. It is probably the most familiar technique for rendering weak phase objects visible. In a chromatographic cell, schlieren measurements are difficult 
because the knife edge reduces an already low transmitted intensity to a level difficult to measure.

The shadow method is the simplest flow visualization procedure. The basic apparatus is just a point light source, a lens and a photographic plate or imaging detector. In the object under investigation, individual light rays are refracted from their original path. If the gradient of the refractive index, $\partial n / \partial y$, is constant along the length of the test section, $l$, then the deflection angle of all rays is the same, and the image plane or photographic plate is uniformly illuminated. Such a density distribution can be represented by a linear wedge of a uniform, transparent medium. However, if the contour of the wedge is curved, the photographic plate will still receive constant illumination corresponding to a density field of constant $\partial^{2} n / \partial y^{2}$. Thus the shadow method visualizes those fields where the second $\partial^{2} n / \partial y^{2}$ is not zero'. The relative intensity change recorded by the detector is given ${ }^{10}$ by eqn. 2 . Eqn. 2 assumes that the detector has a linear response. For photographic film, however, blackening is proportional to the logarithm of incident light intensity, $I$, and eqn. 2 is valid only over a limited range.

$$
\frac{I-I}{I}=\frac{\Delta I}{I}=l \int\left(\frac{\partial^{2}}{\partial x^{2}}+\frac{\partial^{2}}{\partial y^{2}}\right)(\ln n) \mathrm{d} z
$$

\section{EXPERIMENTAL}

Flow visualization measurements were carried out with an apparatus consisting of a pump system, the flow cell, and flow visualization optics. No column was used. The mobile phase was methanol-water (60:40). The samples were aqueous methyl orange or pure water. The flow cell was a Kratos SFA-234, $1 \mathrm{~mm}$ I.D., $1 \mathrm{~mm}$ path length. A single channel piston pump (LDC Milton Roy, Mini-Pump) was used at flow-rate about $2 \mathrm{ml} / \mathrm{min}$. A flat coil pulse dampener (Handy and Harmon) was used to reduce flow fluctuations. Samples of $20 \mu 1$ were injected into the system with a rotary injection valve (Rheodyne 7010).

The shadowgraph apparatus is shown in Fig. 2. A 75-mW Xe arc lamp was used

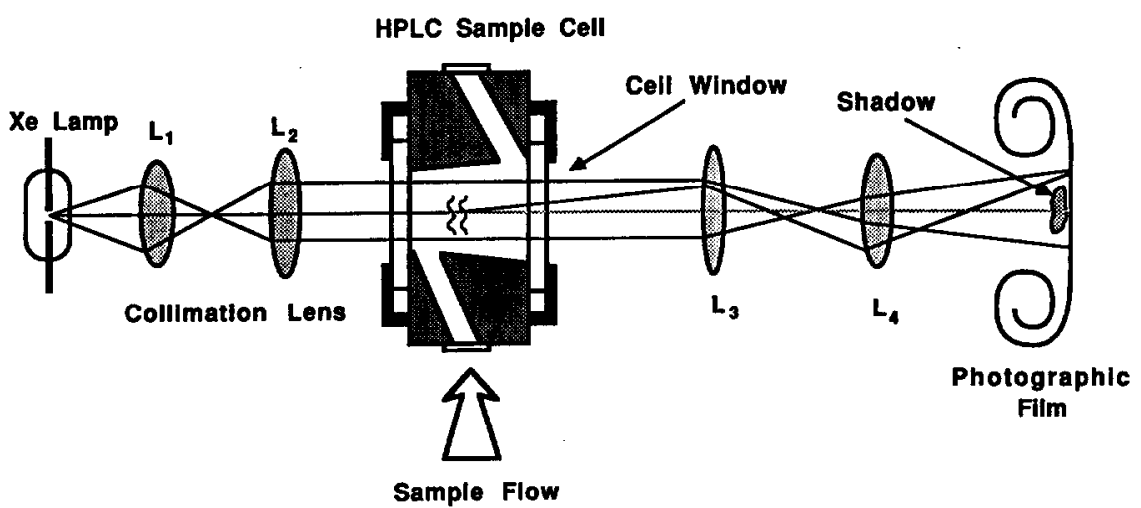

Fig. 2. Shadowgraph apparatus, $\mathrm{L}_{1}, f=25 \mathrm{~mm} ; \mathrm{L}_{2}, f=90 \mathrm{~mm} ; \mathrm{L}_{3}, f=32 \mathrm{~mm} ; \mathrm{L}_{4}, f=3 \mathrm{~mm}$. 
as the point light source. The output from the Xe arc lamp was collimated with lenses of 25 and $90 \mathrm{~mm}$ focal length and directed to the flow cell, which was mounted on translation stages for precise positioning. The images were recorded on Kodak Ektachrome 400 film or Kodak Panatomic X film mounted in a 35-mm SLR camera. In order to enlarge the image from the 1-mm diameter flow cell for photography, two microscope objective lenses of focal length, $f, 32 \mathrm{~mm}$ and $3 \mathrm{~mm}$ respectively, were used. The $3 \mathrm{~mm}$ lens was mounted on a bellows attached to the camera for focusing.

To observe wedge angles among commercial flow cells, a He-Ne laser was directed onto the cell windows and the angle between reflections from entrance and exit windows was measured directly.

\section{RESULTS AND DISCUSSION}

To visualize flow phenomena in a chromatographic cell, the images must be greatly magnified. Preliminary experiments were made with a schlieren apparatus. However, the schlieren method requires blocking of the zero spatial frequency component. This requirement reduced the transmitted light intensity to a level which could not be photographed with apparatus available to us. Thus, the shadowgraph method was used for further experiments.

The shadowgraphs of Fig. 3 show the progress of an aqueous methyl orange sample flowing through the high-performance liquid chromatographic (HPLC) absorbance cell. With pure water as the sample, the progression of shadowgraph images is about the same. The pictures were taken with approximately 2-s intervals between each frame. These images are photographs of color transparencies.

Fig. 3.1 shows the cross section of a HPLC sample cell filled with only mobile phase. Although the mobile phase is moving, it is homogeneous and the flow is not visible.

In Fig. 3.2, methyl orange enters the cell from the bottom. The image shows a bright region in the center and two dark regions on either side. The dark spots are the area where light has been deflected by an inhomogeneous refractive index distribution. The light rays which encounter a small refractive index gradient are deflected but still reach the photographic film. The redistributed intensity is not lost but gives a brighter spot in the center of the image. But some light rays encounter a large refractive index gradient in the opposite direction. They are deflected at large angles and either hit the cell wall or escape detection by the high $f$-number photographic system. The result is that intensity is lost, creating the two dark lobes in the image.

The flow pattern in the cell which gives rise to Fig. 3.2 and the subsequent shadowgraph images, can be qualitatively described. The mobile phase is pumped against the cell window, causing the flow to bifurcate into two streams. The two streams are not exactly equal, so that the shadows are somewhat different on the two sides of the cell. As these two streams continue along the flow path, the shadowgraphs evolve, but always with the presence a two-lobe pattern.

In Fig. 3.3, the sample has flowed through the length of the cell and reached the exit window. At this point the flow has begun to swirl the walls of the cell. Even though the sample has hit the exit window, the upper part of the cell has not yet completely filled with methyl orange. This effect is even clearer in color images made with methyl orange. In these the upper part of the cell is only faintly orange while the bottom is brightly colored. 


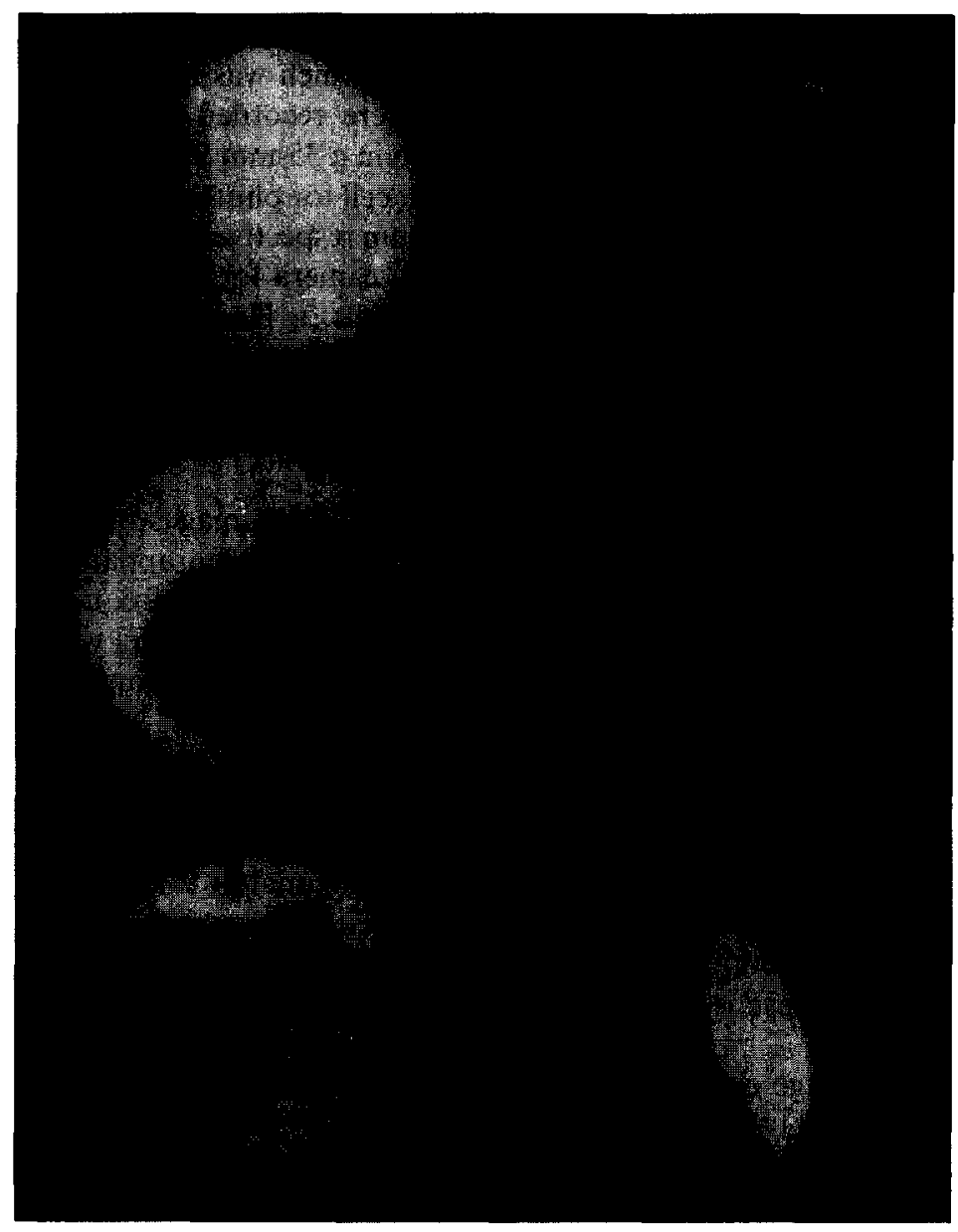

Fig. 3. Shadowgraph images of flow in a liquid chromatographic cell. The sample is aqueous methyl orange in a methanol-water $(60: 40)$ mobile phase. Flow-rate, $2 \mathrm{ml} / \mathrm{min}$. (1) Mobile phase only; (2)-(6) sample through flow cell, at 2-s intervals.

In Fig. 3.4, the sample fills the cell. Part of the sample has already been washed from the cell. But as can be seen from the picture, the fluid in the cell is still not homogeneous. In Fig. 3.5, the tail of the sample flow has entered the cell. Again, the fluid is inhomogeneous. The last image shows the end of the sample flow. Here, mobile phase flows into the cell and generates the bright spot in the center. The remaining sample is distributed along the cell wall deflecting the light and creating shadows. After this the system returns to the uniform intensity distribution of Fig. 3.1.

At no time throughout the evolution of the band, can a homogeneous light intensity be seen. Inhomogeneity occurs because the cell transit time is only $25-30 \mathrm{~ms}$. In the strongly directional flow there is no time for mixing. The constantly changing refractive index distribution becomes a source of detector excess noise. As the band evolves the transmitted intensity wanders around the detector surface, generating small changes in signal caused by local detector response inhomogeneities. 
The shadowgraphs and the flow patterns observed for distilled water are the same as for methyl orange solutions. This observation confirms that the flow pattern is associated with the geometry of the absorbance cell rather than with the nature of the sample. Consequently, the details of the flow patterns will be strongly dependent on the design of the flow cell.

The shadowgraph method does not provide sufficient data to derive a complete description of refractive index distribution in an absorbance cell ${ }^{1,12}$. From eqn. 2 , in order to evaluate the refractive index, $n$, we must perform a double integration of the data. Because the constants of integration are not known it is impossible to obtain the refractive index distribution.

A complete description of the index field is not necessary for evaluation of chromatographic cells. The shadowgraph technique provides information on the spatial redistribution of light at the exit window of the cell. With this information and the projected area of the beam at the detector surface distribution of light on the detector is readily calculated. A description of light intensity distribution on the photodetector surface, rather than of the spatial inhomogeneities within the cell itself, is needed for design of an improved absorbance detector.

The information in the shadowgraphs is sufficient to estimate the distances over which refractive index inhomogeneities extend at the exit window of the cell. Deionized water has a refractive index of 1.3325 at $20^{\circ} \mathrm{C}$ and water-methanol (40:60) mobile phase has a refractive index of 1.3302 at $20^{\circ} \mathrm{C}$. From the small refractive index difference between the mobile phase and deionized water, 0.0023 at $20^{\circ} \mathrm{C}$, a small angular deflection can be assumed. The aperture of the flow cell is $1 \mathrm{~mm}$. Therefore we can estimate from the location of intensity peaks and saddles in the shadowgraphs that inhomogeneities of refractive index extend between 50-100 $\mu \mathrm{m}$ in this LC flow cell.

We measured wedge angles in four different LC cells, which were available in our laboratory. The measurements were made on cells to which no special adjustments had been made. We found that wedge angles varied from $0.5^{\circ}$ to $2^{\circ}$. With careful adjustment, we could reduce the wedge angle to less than $0.06^{\circ}$ in any of the cells. Although our measurements do not constitute a statistically valid study, they do suggest that the windows of a LC flow cell can not be assumed to be parallel unless care is taken in the assembly of the cell.

Even if the front window were aligned perfectly perpendicular to the light path, a wedged cell can cause the baseline to drift. Eqn. 1 shows that at normal incidence, $\theta_{\mathrm{i}}=0$, the deviation angle $\delta \approx \alpha(n-1)$ and

$$
\frac{\mathrm{d} \delta}{\mathrm{d} n}=\alpha
$$

For a cell wedged at $0.5^{\circ}$, a refractive index change of $10^{-3}$ unit can cause the light to deviate $0.0005^{\circ}$. This effect can be important if the mobile phase is not mixed well or in gradient elution. Because photodiodes have different responses from point to point, this beam deviation is a further, if small, source of noise in absorbance detection.

We can estimate the temperature rise, $\Delta T$, in the cell caused by light absorption. Using the equation, $\Delta T=(P \alpha l / J) / k 2 \pi w l$ where $k$ is thermal conductivity, $\alpha$ is absorptivity, $l$, absorption length, $w$, beam radius, $J=4.18 \mathrm{~J} /$ cal is Joule's constant and assuming $0.1 \mathrm{~mW}$ power, $P$, delivered to the cell, and absorbance $1 \cdot 10^{-5}$, we 
calculate the temperature rise in a flow cell to be on the order of $10^{-6}-10^{-8}$ degree. For most compounds $\mathrm{d} n / \mathrm{d} T$ is approximately $10^{-4}$, so that $\Delta n$ is about $10^{-10}$. The thermal lens effect is probably not a significant noise source.

Eliminating the noise sources associated with flow in a LC absorbance cell appears to be a formidable problem. There is no simple design which eliminates boundary layers and provides sufficient mixing to generate completely uniform flow. Because flow cell length and diameter are the same the mixing time must always be greater than the nominal transit time. To remove the problems associated with wedged cell windows, one must align cell windows carefully. To be certain that thermal effects are negligible, the cell and the detector must be designed to dissipate heat rapidly and uniformly. Compensation for these problems appears to be an attractive alternative to attempts at their complete elimination.

Pang and Morris ${ }^{13}$ proposed the use of a retroreflective array as an approximate phase conjugator capable of compensating for the problems identified in this communication. They described an absorbance detector configured with a retroreflective array in the phase conjugation configuration. The system provided dynamic compensation of refractive artifacts, and, in addition, doubled the measured absorbances. The signal-to-noise ratio was increased overall by a factor of 4 to 6 . A similar system was shown to provide at least a ten-fold reduction of noise in a flame atomic absorption system ${ }^{14}$.

Pang and Morris ${ }^{13}$ used a commercial array with elements spaced on $0.16 \mathrm{~mm}$ centers. Their work was carried out before detailed information about the actual non-idealities of a chromatographic flow cell, or even detailed knowledge of the optical properties of the array ${ }^{15}$ was available. Our observations demonstrate that the Pang and Morris array is too coarse for optimum compensation for refractive index gradients in a chromatographic flow cell. Better results might be expected if arrays of 20-50 elements/mm are employed. Arrays with this element spacing are difficult to fabricate. Alternatively, beam-expanding optics might be used to magnify the exit at least five times and roughly collimate it for efficient operation of the array. Performance of detectors using this compensation scheme will be reported in a subsequent communication.

\section{ACKNOWLEDGEMENTS}

The authors wish to thank Timothy Nevius (Anspec, Ann Arbor, MI, U.S.A.) for helpful discussions. Financial support was provided by Anspec through a National Institutes of Health Small Business Innovative Research Grant.

\section{REFERENCES}

1 E. Yeung (Editor), Detectors for Liquid Chromatography, Wiley, New York, 1986.

2 S. R. Abbot and J. Tusa, J. Liq. Chromatogr., 6 (1983) 77-104.

3 P. Jandera and Churáček, Gradient Elution in Column Liquid Chromatography, Theory and Practice, Elsevier, Amsterdam, 1985.

4 J. N. Little and G. J. Fallick, J. Chromatogr., 112 (1975) 389-397.

5 J. E. Stewart, Appl. Opt., 18 (1979) 5-6.

6 J. E. Stewart, Appl. Opt., 20 (1981) 654-659.

7 Photodiode Catalog, EG\& G Photon Devices, Salem, MA, 1986, p. 42.

8 E. Hecht and A. Zajac, Optics, Addison-Wesley, Reading, MA, 1979. 
9 W. Merzkirch, Flow Visualization, Academic Press, New York, 1974.

10 F. J. Weyl, in R. W. Ladenburg (Editor), Analysis of Optical Methods (Physical Measurements in Gas Dynamics and Combustion, Vol. 9), Princeton University Press, Princeton, NJ, 1954.

11 L. S. Taylor, AIAA J., 8 (1970) 1284-1287.

12 M. S. Uberoi and L. S. G. Kovasznay, J. Appl. Phys., 26 (1955) 19-24.

13 T.-K. J. Pang and M. D. Morris, Anal. Chem., 57 (1985) 2700-2701.

14 Y.-Z. Hsieh, E. T. Johnson, R. D. Sacks and M. D. Morris, Appl. Spectrosc., 41 (1987) 84-88.

15 K. Peck and M. D. Morris, Rev. Sci. Instrum., 58 (1987) 189-196. 